\title{
On the velocity of ghost particles and the bias errors in Tomographic-PIV
}

\author{
G. E. Elsinga $\cdot$ J. Westerweel $\cdot$ F. Scarano • \\ M. Novara
}

Received: 17 December 2009/Revised: 2 June 2010/Accepted: 26 June 2010/Published online: 14 July 2010

(C) The Author(s) 2010. This article is published with open access at Springerlink.com

\begin{abstract}
The paper discusses bias errors introduced in Tomographic-PIV velocity measurements by the coherent motion of ghost particles under some circumstances. It occurs when a ghost particle is formed from the same set of actual particles in both reconstructed volumes used in the cross-correlation analysis. The displacement of the resulting ghost particle pair is approximately the average displacement of the set of associated actual particles. The effect is further quantified in a theoretical analysis and in numerical simulations and illustrated in an actual experiment. It is shown that the bias error does not significantly affect the measured flow topology as deduced in an evaluation of the local velocity gradients. Instead, it leads to a systematic underestimation of the measured particle displacement gradient magnitude. This phenomenon is alleviated when the difference between particles displacement along the volume depth is increased beyond a particle image diameter, or when the reconstruction quality is increased or when the accuracy of the tomographic reconstruction is improved. Furthermore, guidelines to detect and avoid such bias errors are proposed.
\end{abstract}

\section{Introduction}

Tomographic-PIV is a recent technique for measuring the three-dimensional instantaneous velocity distribution in a

G. E. Elsinga ( $₫) \cdot$ J. Westerweel

Laboratory for Aero and Hydrodynamics,

Department of Mechanical Engineering,

Delft University of Technology,

Delft, The Netherlands

e-mail: g.e.elsinga@tudelft.nl

F. Scarano $\cdot$ M. Novara

Aerodynamics Group, Department of Aerospace Engineering,

Delft University of Technology, Delft, The Netherlands fluid (Elsinga et al. 2006a). In this method, images of the tracer particles are recorded from several viewing directions simultaneously, after which a tomographic reconstruction algorithm is used to obtain the three-dimensional light intensity distribution associated to the tracer particle distribution within the measurement volume. The particle displacement field between subsequent recordings, hence velocity, then results from a cross-correlation analysis of two reconstructed light volumes analogue to planar PIV (Adrian 1991). Compared to other existing 3-D PIV methods, such as 3-D particle tracking (Maas et al. 1993) and digital holographic-PIV (Coëtmellec et al. 2001), Tomographic-PIV can operate at higher seeding densities, typically 0.05 particles per image pixel (Elsinga et al. 2006a), which is advantageous as this allows for a finer spatial resolution of the measurement. As a result, it is now being applied as a useful tool in fluid dynamics investigations of predominantly turbulent flows (e.g. Elsinga et al. 2007, 2010; Elsinga and Marusic 2010; Schröder et al. 2008a, b; Hain et al. 2008; Humble et al. 2009; Atkinson et al. 2009; Kühn et al. 2009; Ortiz-Duenas et al. 2010). Where the mean and RMS flow statistics have been evaluated in these experiments and compared against other data, they appear to agree to within approximately 0.3 pixel particle displacement (Elsinga 2008) showing that the method can be accurate.

Although the reported accuracy of the TomographicPIV technique is hopeful, very little is known about possible velocity bias errors that may appear when the experimental conditions are changed. Here, a distinction needs to be made between the errors introduced in the imaging and cross-correlation steps of the experimental procedure, and the errors coming from the tomographic reconstruction. The former are in common with planar PIV and have already been documented (for an overview see 
Raffel et al. 1998 or Adrian and Westerweel 2010), while the latter are yet to be investigated. What is known is that the tomographic reconstructions contain spurious light intensity peaks, which are referred to as ghost particles (Elsinga et al. 2006b). Their origin will be discussed in more detail in Sect. 2.

Surprisingly, first results from previous TomographicPIV measurements in a cylinder wake have indicated that the accuracy of the velocity vectors shows little to no dependence on the accuracy of the tomographic particle reconstruction, that is the number of ghost particles compared to the number of actual particles (Elsinga et al. 2006b; Elsinga 2008). This independence of the velocity and reconstruction accuracy applies at least within the experimental parameter range considered in that particular cylinder wake experiment, which included, however, a case where the ghost particles outnumbered the actual particles. Under that condition one would expect the falsely reconstructed particles to dominate the measurement and introduce errors in the particle displacement obtained by cross-correlation. This behaviour is clearly not observed in the actual measurements, which further illustrates the need for a better understanding of the ghost particle phenomenon.

In this paper, we will present a model describing the ghosts' contribution to the velocity measurement (Sect. 3) and provide supporting evidence for it from numerical simulations and experiment (Sects. 4-5). It will be shown that ghosts can introduce an important velocity bias error under some circumstances. Further insight into the crosscorrelation signal produced by the ghosts will be obtained, which enables us to formulate criteria and experimental procedures directed at preventing ghost particles from affecting the accuracy of the velocity field.

The present model builds on previous discussions of ghost particles in 3-D particle tracking (Maas et al. 1993) and aims at using the experimental parameters typically available at the design stage of an experiment. Alternative to our engineering approach, a rigorous mathematical treatment of ghost formation can be found in the literature describing the influence of the Null space in tomographic reconstructions (e.g. Louis 1981; Natterer 1986; Munshi 2002). These general works, however, are not specifically targeted at particles, nor do they contain information on ghost velocities.

\section{Formation of ghost particles}

The formation of ghost particles, or reconstruction noise, is illustrated in Fig. 1 for a 2-camera system. The measurement volume contains two particles, which are seen by camera 1 along the lines-of-sight $L O S_{1}$ and $L O S_{2}$ and by camera 2 along the lines $L O S_{3}$ and $L O S_{4}$ resulting in the

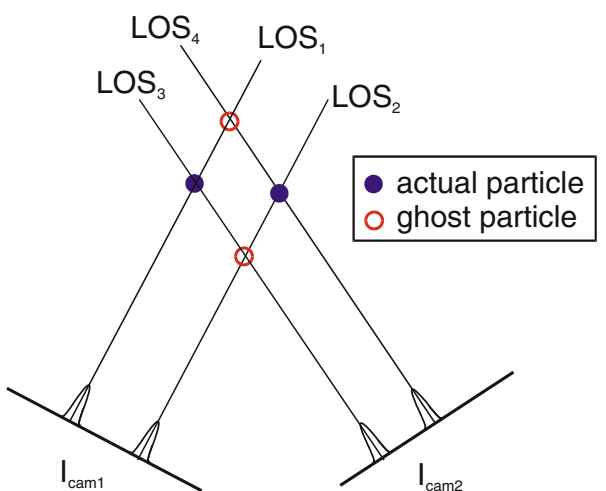

Fig. 1 Formation of ghost particles in a 2-camera setup

recorded images $I_{\mathrm{cam} 1}$ and $I_{\mathrm{cam} 2}$. In an actual measurement, these images are used to reconstruct the particle distribution in the measurement volume. Reconstructed particles may be formed at the intersections of a lines-of-sight from each camera corresponding to a particle (i.e. $\operatorname{LOS} 1-4$ ). The lines triangulate in four points in the present example resulting in three possible solutions for the particle reconstruction (Fig. 2). It can be shown that starting from an initially uniform intensity distribution the present MART tomographic reconstruction algorithm produces an intensity field corresponding to the 4-particle solution shown in Fig. 2-right, where the particles have equal intensity. Two of the reconstructed particles correspond to actual tracer particle and two are ghost particle or noise peaks in the reconstruction (Fig. 1).

In the general case of an $\mathrm{N}$-camera system, a particle (either ghost or actual particle) is reconstructed in a point in the volume, when each of the $N$ lines-of-sight going through that point corresponds to a particle image in the respective $N$ recordings. This correspondence needs to be within a particle image diameter. Assuming that ghost particle formation can be described as a random process and ignoring the iterative corrections in the tomographic reconstruction, the chance that a given voxel unit volume (or a point) corresponds to a particle image in each of the $N$ recordings, hence results in a ghost, is $\left(p p p \cdot A_{\mathrm{p}}\right)^{N}$, where ppp (particles per pixel area) is the particle image density in the recordings and $A_{\mathrm{p}}$ is an effective particle image area in pixel units. It should be noted that strictly speaking the
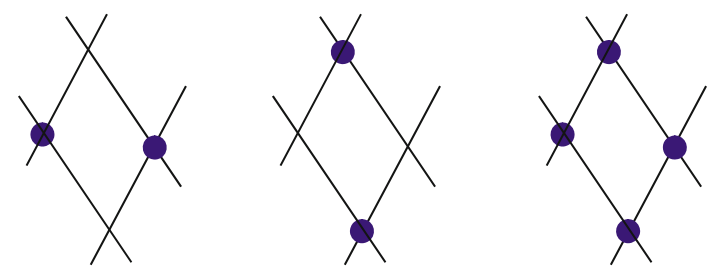

Fig. 2 Possible reconstruction solutions to the 2-particle-2-camera problem of Fig. 1 
ghosts depend on the actual particle distribution due to the MART iterations, which converge to the so-called maximum entropy solution (Herman and Lent 1976). However, Atkinson and Soria (2009) have found that the ghosts produced by their initial MLOS reconstruction technique (which in essence is very similar to the simplified reconstruction process used in this analysis of ghost formation) persist after subsequent MART iterations, but they may decrease in peak intensity. This suggests that the present random-process approach is appropriate. Further, the effective area $A_{\mathrm{p}}$ is not necessarily equal to the total image area based on the diameter of the diffraction spot, but can be smaller because triangulation of the low image intensity near the edge of the diffraction spot creates a ghost of insignificant intensity. Then, the expected total number of ghost particles $N_{\mathrm{g}}$ in the measurement volume is given by:

$N_{\mathrm{g}}=\left(p p p \cdot A_{\mathrm{p}}\right)^{N} \cdot l_{x} \cdot l_{y} \cdot l_{z}$

where $l_{x} \cdot l_{y} \cdot l_{z}$ are the dimensions of the reconstruction volume in $x$-, $y$ - and $z$-direction in voxel length units (width, height, and depth). The number of actual tracer particles in the volume $N_{\mathrm{p}}$ can be estimated from the particle image density on the recordings as:

$N_{\mathrm{p}}=p p p \cdot l_{x} \cdot l_{y}$

For sake of simplicity, it is assumed here that the pixels and the voxels projected on the recordings have equal dimensions so that the entire volume can be imaged onto a $l_{x} \cdot l_{y}$ pixel area. The assumption holds reasonably well in real experiments considering that the depth of the volume, $l_{z}$, usually is much smaller than its height and width. Then, an indicator of the signal-to-noise ratio in the tomographic reconstruction of the $3 \mathrm{D}$ particle distribution is chosen as the ratio of the number of actual tracer particles and the number of ghost particles $N_{\mathrm{p}} / N_{\mathrm{g}}$ :

$\frac{N_{\mathrm{p}}}{N_{\mathrm{g}}}=\frac{1}{p p p^{N-1} \cdot A_{\mathrm{p}}^{N} \cdot l_{z}}$.

It should, however, be kept in mind that this ratio alone does not account for the effect of the different peak intensity between ghosts and actual particles as previously discussed by the author (Elsinga et al. 2006a, 2008, histograms reveal a lower peak intensity for ghosts compared to the actual particles). In this particular case, the results will yield a conclusion from a conservative point of view whereby ghost and actual particles have the same peak intensity. Nevertheless, the mechanism under scrutiny is captured in its general features. From Eq. 3, it is readily seen that the signal-to-noise ratio in the reconstruction strongly depends on the particle image density expressed in ppp. With decreasing $p p p$ the occurrence of random intersections of lines-of-sight corresponding to particle images rapidly decreases, hence the number of ghost particles drops. Furthermore, the reconstruction signal-tonoise ratio increases with the number of cameras $N$, because $p p p<1$. The ratio $N_{\mathrm{p}} / N_{\mathrm{g}}$ furthermore depends on the thickness of the reconstruction volume, which is proportional to the interception length of the line-of-sight with the volume. Finally, the effective particle image area $A_{\mathrm{p}}$ is usually a constant in a given experimental setup, where the particles are in focus and their images are diffraction limited. The effects of the camera viewing angles are generally weak within the range commonly used in experiments (Elsinga et al. 2006a), and they are neglected in the present discussion. Camera calibration errors are also not taken into account, as they can be reduced to a fraction of a pixel by a volume self-calibration (Wieneke 2008).

The relation of Eq. 3 has been supported by results from 4-camera Tomographic-PIV experiments in which the particle image density and volume thickness varied (Elsinga et al. 2006b; Elsinga 2008; Michaelis and Wieneke 2008). In these studies, the effective particle image area $A_{\mathrm{p}}$ was obtained from a fit of Eq. 3 on the available reconstruction noise data indicating typical values around 2.5-3.0 pixel area in agreement with the individual particles images observed in the recordings. The reconstruction noise in these experiments was estimated by the ratio of the reconstructed light intensity inside and outside the light sheet (Michaelis and Wieneke 2008) or by the ratio of the number of reconstructed intensity peaks in these respective regions (Elsinga et al. 2006b).

\section{Ghost particle pairs}

To assess the contribution of the ghost particles to the velocity measurement, not only their occurrence in individual volumes needs to be considered, but also the way in which they add to the signal in the cross-correlation map. This is a problem of ghost reoccurrence in both reconstructed volumes. A model for the ghost particle displacement, or velocity, is offered in Fig. 3. In this example, a ghost particle is formed from 4 actual particles in a 4-camera system. In case the displacement normal to the viewing direction between the two exposures is nearly equal for all 4 actual particles (to within approximately one particle image diameter, Fig. 3-left), the ghost particle is formed in both exposures from the same 4 actual particles and is displaced roughly by the average displacement of these 4 actual particles. The resulting ghost particle pair will then affect the correlation map. Because the physical location of the ghost pair is different from that of the actual particles, the ghost pairs can spread the actual velocity information out over the entire volume in principle. This has a smoothing effect on the measured velocity fields 


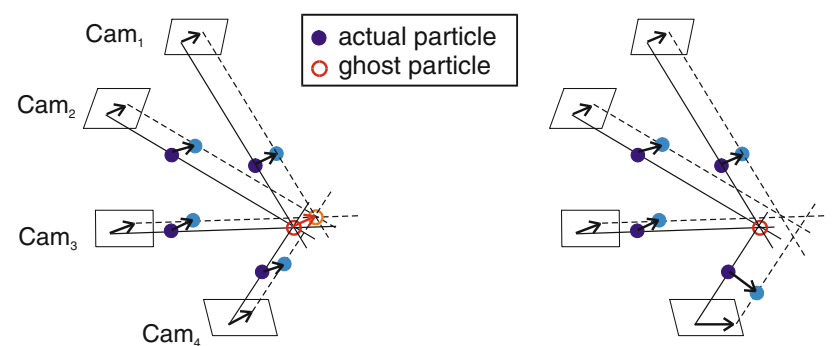

Fig. 3 Formation of ghost particles that contribute to the crosscorrelation (left) and non-correlating ghost particle (right). Dark particles and solid lines-of-sight correspond to the first exposure. Bright particles and dashed lines-of-sight correspond to the second exposure

resulting in the reduction of velocity gradients. It can also be viewed as a velocity modulation. If, however, the displacement from a single one of these 4 actual particles (the particle corresponding to camera 4 in Fig. 3-right) is different from the others, the lines-of-sight in the second exposure (dashed lines) no longer intersect in a single common point. Hence, the ghost particle is returned in only one of the exposures, which does not contribute to the build up of a (false) correlation peak. Please note that variations in particle displacement normal to the viewing direction are considered and that displacement variations in the viewing direction do not change the recorded images hence do not affect ghost formation. In contrast, a displacement variation of the actual particles normal to the viewing direction relates directly to the particle image positions and their corresponding lines-of-sight, hence the triangulation of ghost particles. Particularly when the displacement in this direction varies by more than a particle image diameter, a ghost may be formed in one exposure but not in the other (from the same set of actual particles).

The proposed mechanism has some important consequences. First, a velocity may be detected outside the illuminated domain related to the ghost particle pairs outside the light sheet. Whereas actual particles are reconstructed only within the laser sheet, ghosts formation is a random process that takes place throughout the entire reconstructed volume. Since the reconstructed volume is usually slightly larger than the laser sheet, the velocity can be affected by ghosts in some regions near the edges of the reconstructed volume (Elsinga et al. 2006b). There is, however, a simple remedy to this issue: detect the light sheet in reconstructed light intensity volumes (e.g. Elsinga et al. 2006a), so that the light position is known exactly and velocity vectors outside can be ignored. A second, more concerning consequence is the velocity modulation by the reoccurring ghost particles to which there is no straightforward solution. Therefore, this aspect will be studied further in the following section by means of numerical simulations.
Based on the presented model for the ghost velocity, a new estimate for the ratio of actual particles over ghost particles can be formulated, in which only the reoccurring ghosts $N_{\mathrm{g}}^{*}$ are considered. As discussed earlier, these ghost pairs are formed from a set of $N$ actual particles that are displaced coherently between frames, where $N$ is the number of views, i.e. number of cameras. This occurs when the difference in displacement normal to the viewing direction among the set of actual particles is smaller than a particle image diameter $d_{\mathrm{p}}$, approximately. Further simplification can be made by assuming only velocity variations along the volume depth (the present $z$-direction) are relevant, which is justified by the limited range of viewing angles and by the relatively small volume thicknesses used to Tomographic-PIV experiments. Then, the distance in depth direction over which the actual particle displacement is coherent, is given by $l^{*}$, which will be normalized by the total reconstructed volume thickness $l_{z}$. For a ghost particle pair to occur, it is necessary that the $N$ actual particles from which it is formed must all lie within this $l * / l_{z}$ 'slice' of the volume, which effectively reduces the relevant particle image density in the recordings to $p p p \cdot l * / l_{z}$. Consequently, the chance of a reoccurring ghost at any given point in the volume is $\left(p p p \cdot A_{\mathrm{p}} \cdot \frac{l^{*}}{l_{z}}\right)^{N}$, compared to $\left(p p p \cdot A_{\mathrm{p}}\right)^{N}$ when considering all particle images as before in Sect. 2. For the total number of reoccurring ghosts in the volume this results in:

$N_{g}^{*}=\left(p p p \cdot A_{\mathrm{p}} \cdot \frac{l^{*}}{l_{z}}\right)^{N} \cdot l_{x} \cdot l_{y} \cdot l_{z}$

Combining $N_{\mathrm{g}}$ * with the total number of actual particles $N_{\mathrm{p}}$ (Eq. 2) yields the new ratio:

$\frac{N_{\mathrm{p}}}{N_{\mathrm{g}}^{*}}=\frac{1}{p p p^{N-1} \cdot\left(A_{\mathrm{p}} \cdot \frac{l^{*}}{l_{z}}\right)^{N} \cdot l_{z}}$

The length scale $l^{*}$ depends on the actual velocity field to be measured and hence has been defined loosely so far as the volume thickness over which the actual particle displacement normal to the viewing direction changes by no more than the particle image diameter. It may still vary with the $x$ and $y$ location in the volume (say the across the field of view). As seen from Eq. 5, the relative number of reoccurring ghosts strongly depends on $l^{*}$ and therefore an attempt will be made in the following section to specify the relation between the velocity field and $l *$ for two different flow cases.

\section{Numerical simulations}

Now the relevant parameters in ghost particle pair formation have been identified, their impact on the velocity 
measurement will be demonstrated by means of numerical simulations of a Tomographic-PIV experiment. Two flow cases will be considered here: a shear layer representing boundary layer and mixing layer flow, and an array of counter rotating vortices resembling the secondary flow structures in a separated wake.

The layout of the numerical simulations follows those previously proposed by Elsinga et al. (2006a). Particles are distributed in a 2 -D , $1,000 \times 200$ voxel slice of the volume, and they are imaged by four 1-D pixel arrays, representing a four-camera imaging system. The particle image diameter is 3 pixels. A coordinate system is defined in which $z$ is the volume depth coordinate and $x$ is the direction along the front of the volume slice. The viewing directions of the cameras with respect to the $z$-axis are $-30^{\circ},-15^{\circ}, 15^{\circ}$ and $30^{\circ}$. Between the exposures the particles are displaced according to a predefined velocity distribution. Then the volume intensity distribution is reconstructed from these images using the MART algorithm with 5 iterations (Elsinga et al. 2006a) or alternatively the single pass multiplicative line-of-sight (MLOS) method (as proposed by Worth and Nickels 2008; Atkinson and Soria 2009). Like before (Elsinga et al. 2006a), the resulting reconstructed particle intensity fields $E_{1}$ can be compared with the so-called ideal reconstruction $E_{O}$, which is taken as Gaussian intensity blobs at the actual particle positions. The comparison is quantified by the reconstruction quality factor $Q$ defined as:

$$
Q=\frac{\sum_{x, z} E_{1}(x, z) \cdot E_{0}(x, z)}{\sqrt{\sum_{x, z} E_{1}^{2}(x, z) \cdot \sum_{x, z} E_{0}^{2}(x, z)}}
$$

Furthermore, by removing the actual particles from the MART reconstruction, an intensity distribution consisting only of contributions from ghost particles can be created, which enables a separate analysis of their contribution to the velocity measurement.

A 2-D ensemble cross-correlation analysis (Meinhart et al. 2000) combined with iterative multi-grid and window deformation (Scarano and Riethmuller 2000) is performed of the MART, MLOS, ideal reconstructions and the ghosts to obtain the particle displacement in each of these volume intensity fields. The final correlation window size is $31 \times 31$ voxels and each ensemble contains 31 volume slices, which resembles a $31^{3}$ correlation volumes in case of a real 3-D measurement. Finally, the velocity fields are averaged over four independent realizations of such correlation averaging results to suppress correlation noise.

In present simulations, the particle image density is varied as well as the predefined actual particle displacement field, which relate to the parameters $p p p$ and $l * / l_{z}$ in Eq. 5, respectively. The effects of the other parameters in Eq. 5 is ignored here, as $A_{\mathrm{p}}$ does not vary significantly among experiments (it is usually defined by the depth of focus constraint) and $l_{z}$ has a relatively weak effect on the results compared to the other parameters. It should be stressed that the quoted $p p p$ in this section is that of a socalled 1-D image, which can be thought of as an individual pixel line extracted from a regular 2-D recording. The 1-D ppp can be converted to a particle image density in the full 2-D images by considering that each particle image in the 2-D recording extends over three, i.e. the particle image diameter, (1-D) pixel lines. Consequently, each pixel line also intercepts all particles in the two adjacent lines and hence the 1-D ppp is three times the $p p p$ in a 2-D image. This conversion is actually the same as keeping the source density (Adrian 1991), $N s=p p p \cdot A_{\mathrm{p}}$, constant with $A_{\mathrm{p}}=$ $3 \times 1$ pixel area in the $1-\mathrm{D}$ pixel array and $A_{\mathrm{p}}=3 \times$ 3 pixel area in regular 2-D images. An overview of the used particle image densities with their MART reconstruction quality factor and their corresponding 2-D particle image densities is given in Table 1.

As mentioned, the present 1-D images can be regarded as a pixel line taken from the full 2-D recordings. Similarly, the reconstruction here represents a slice out of the complete 3-D volume. Such conditions may occur in actual experiments where the cameras are all positioned along a single line and placed at a large distance. Then, the magnification over the volume depth may be approximated as constant and the lines-of-sight from each camera become almost parallel. This appears a reasonable assumption, as these parameters are changing very gradually over the measurement volume, commonly. Nevertheless, most Tomographic-PIV setups feature a rectangular camera arrangement, not a linear one. This is likely to have a minor effect on the reconstruction noise levels comparable to changing the cameras viewing directions. The effect remains to be quantified, but a previous fully 3-D simulation using a rectangular arrangement did yield a reconstruction quality $Q$ comparable to the linear camera arrangement (Elsinga et al. 2006a).

\subsection{Shear layer}

The imposed particle displacement is a linear function of the depth coordinate, representative of a shear layer. The motion field is given by:

$\Delta x=5+\alpha z$

where $\alpha=(\mathrm{d} u / \mathrm{d} z) \Delta t$ is the displacement gradient determining the actual particle displacement variation over the volume depth, $z$ is the volume depth coordinate of the particle (in voxel length units) and $x$ is the particle coordinate (in voxel lengths) in the homogeneous direction along the front of the volume slice. The particle displacement along the depth direction $z$ is always zero. 
Table 1 Overview of the particle image densities used in the simulations
Fig. 4 The average particle displacement over the volume depth in a numerical simulation for displacement gradients $\alpha=0.01,0.02,0.03$, and 0.05 voxels/voxel, and $p p p_{1-\mathrm{D}}=0.15$. Results are presented for MART, MLOS, and ideal reconstructions together with the ghost particle velocity and they indicate a velocity gradient modulation caused by ghost particles, which decreases as the displacement gradient increases

\begin{tabular}{llll}
\hline $\begin{array}{l}\text { Source density } \\
N s=p p p \cdot A_{\mathrm{p}}\end{array}$ & $\begin{array}{l}\text { ppp in 1-D images } \\
\left(A_{\mathrm{p}}=3 \times 1 \text { pixels }\right)\end{array}$ & $\begin{array}{l}\text { Corresponding } p p p \text { in } \\
\text { 2-D images }\left(A_{\mathrm{p}}=3 \times 3 \text { pixels }\right)\end{array}$ & $\begin{array}{l}\text { MART reconstruction } \\
\text { quality factor } Q\end{array}$ \\
\hline 0.15 & 0.05 & 0.017 & 0.95 \\
0.30 & 0.10 & 0.033 & 0.87 \\
0.45 & 0.15 & 0.05 & 0.74 \\
0.90 & 0.30 & 0.10 & 0.52 \\
1.50 & 0.50 & 0.17 & 0.43 \\
\hline
\end{tabular}
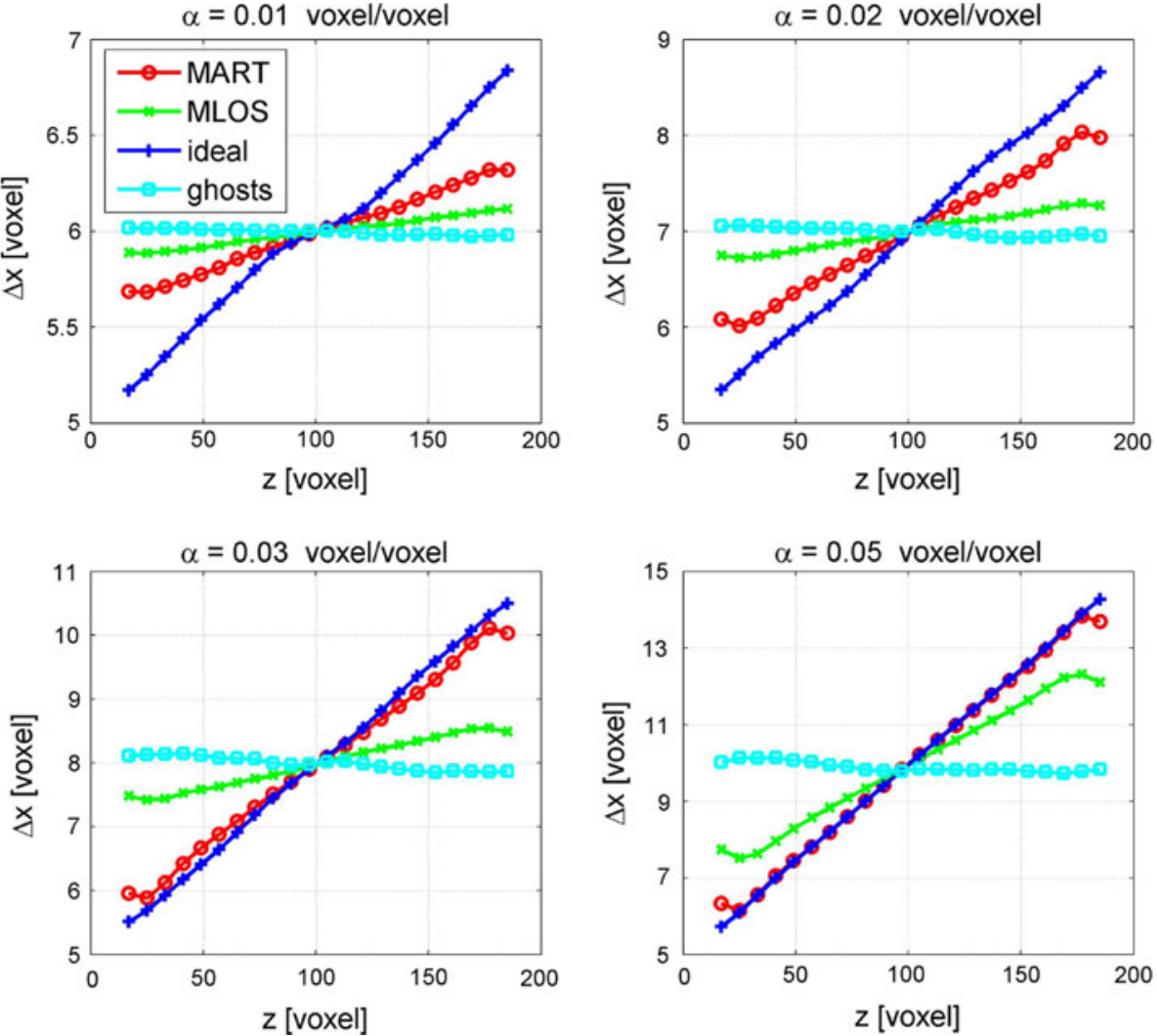

To assess the effect of different gradients $\alpha$ and the other experimental parameters on the velocity measurement, Fig. 4 presents results obtained for a 4-camera system with a (1-D) particle image density of $0.15 \mathrm{ppp}$ showing average velocity profiles along the depth coordinate $z$. The reconstruction quality factor $Q$ is 0.75 for these conditions, which previously has been considered to be the lower limit for reconstruction accuracy (Elsinga et al. 2006a). These results clearly show a systematic underestimation of the velocity gradient consistent with the proposed model for the ghost particle velocities. As $\alpha$ is increased, hence the actual particle displacement variation along $z$, this modulation effect decreases, which happens at a faster rate for MART compared to the MLOS reconstruction. The MART and MLOS results also reveal a kink at either end of each profile, which are edge effects and not relevant to the discussion here. Additionally, a small discrepancy may be noticed between the input velocity and the particle displacement distribution obtained from the ideal reconstruction (especially for $\alpha=0.01$ ), which must be attributed to a bias introduced by the cross-correlation algorithm. Further, as predicted, the ghost particle displacement is the depth average of the actual displacement throughout the volume. Loss of ghost particle correlation is also evident from a decreasing cross-correlation signal-to-noise ratio with increasing $\alpha$ in the analysis of the ghost-only volumes (Fig. 5). This signal-to-noise ratio has been defined here as the ratio of the first and second correlation peak.

The effect of the ghosts on the cross-correlation is further illustrated by the average correlation maps (Fig. 6), where averaging has been performed in the homogeneous $x$-direction. Cross-plots through the correlation peak (Fig. 7) reveal that the correlation map for a MART reconstruction can be considered as the correlation map of an ideal reconstruction combined with the map from the ghost-only field, which shows that the ghost particle 
Fig. 5 The average crosscorrelation signal-to-noise ratio over the volume depth in a numerical simulation for displacement gradients $\alpha=0.01,0.02,0.03$ and 0.05 voxels/voxel, and $p p p_{1-\mathrm{D}}=0.15$. Results are presented for MART, MLOS, ideal reconstructions and ghost particles and they indicate a de-correlation of the ghost particles with increasing displacement gradient
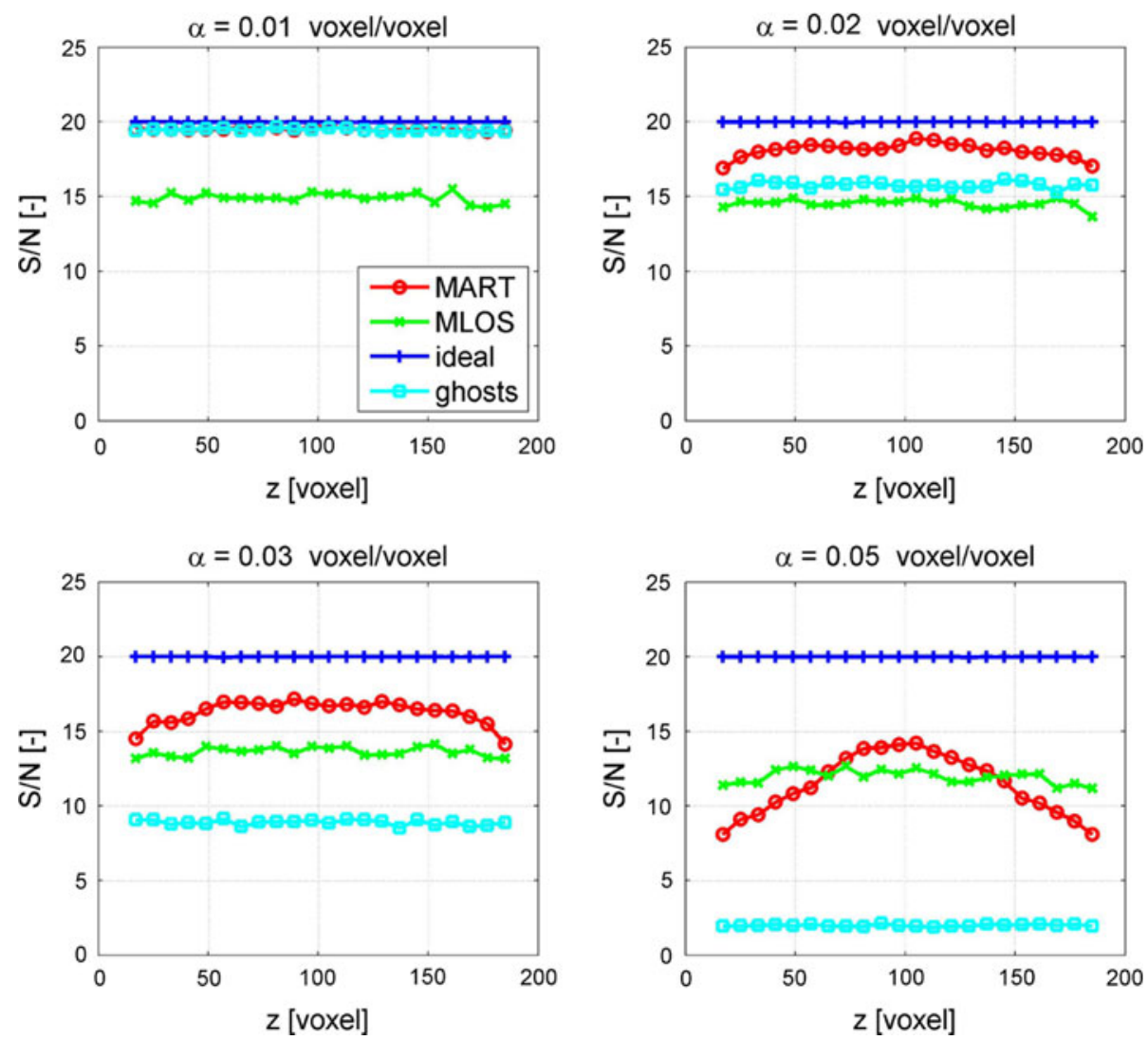

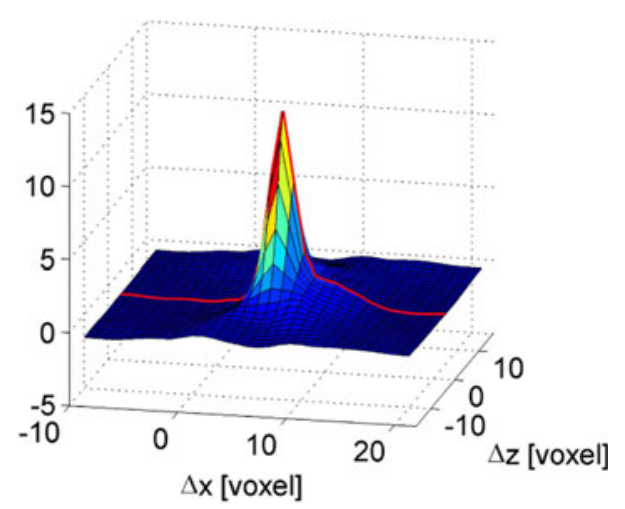

Fig. 6 Example of an average cross-correlation map from a MART reconstruction. The average is taken over several maps at the same $z$ location. The red line indicates the profile through the correlation peak at zero displacement in depth direction $(\Delta z=0)$, which is presented in more detail in Fig. 7

distribution is largely independent of the actual particle distributions (uncorrelated). This observation supports the assumption in Sects. 2 and 3 that the creation of ghosts can be described as a random process. It also allows a separate discussion of the ghost and actual particles contributions to the cross-correlation maps. The latter produce a single well-defined peak at the actual particle displacement, which is illustrated for two depth position, one near the edge of the volume at $z=25$ voxels (Fig. 7-left) and one near the volume centre at $z=95$ voxels (Fig. 7-right). In contrast, the ghosts produce a broad peak that is the same throughout the volume (compare Fig. 7-left with 7-right) and covers the full range of actual particle displacements over the volume depth. Given the linear displacement profile considered here, the maximum is attained at the depth-averaged actual displacement. For a relatively high displacement gradient ( $\alpha=0.05$ voxels/voxel, Fig. 7-top), the ghosts peak ranges from 5 to 15 voxels displacement, which is the range of actual particle displacements inside this volume, with the correlation maximum in the middle at 10 voxels displacement, which is the depth-averaged actual displacement. Note that the ghosts average correlation map does not depend on the depth position $z$ for a constant displacement gradient $\alpha$. Only the peak from the actual particles varies with $z$. For smaller gradients (e.g. $\alpha=$ 0.01 voxels/voxel, Fig. 7-bottom), the range of displacements in the volume is smaller and consequently the ghost correlation peak narrows. Because the area under the ghosts' peak is representative of the number of ghosts in the volume and the ghost intensity, which are both independent of the actual displacement, the ghosts correlation peak value increases with decreasing $\alpha$. As a result, the relative importance of the ghosts in the correlation map increases resulting in a larger bias error as observed in the velocity profiles (Fig. 4). 
Fig. 7 Profiles through the peak in the averaged crosscorrelation map for the MART reconstruction, ideal reconstruction and ghosts at $z=25$ voxels near the border (left) and at 97 voxels near the centre of the volume (right).

The top and bottom row present results for displacement gradients of 0.05 and 0.01 voxels/voxel, respectively. The peak width for the ghost is approximately equal to the range of actual particle displacements in the volume with the maximum at the average displacement of the actual particles. The profiles indicate that the MART correlation map is a combination of the maps for the actual particles (i.e. the ideal reconstruction) and the ghosts

Fig. 8 Modulation of the displacement gradient versus displacement gradient $\alpha$ for varying 1 -D particle image densities. Modulation is defined as the measured divided by the actual displacement gradient. Results are presented for MART (left) and MLOS (right) reconstructions
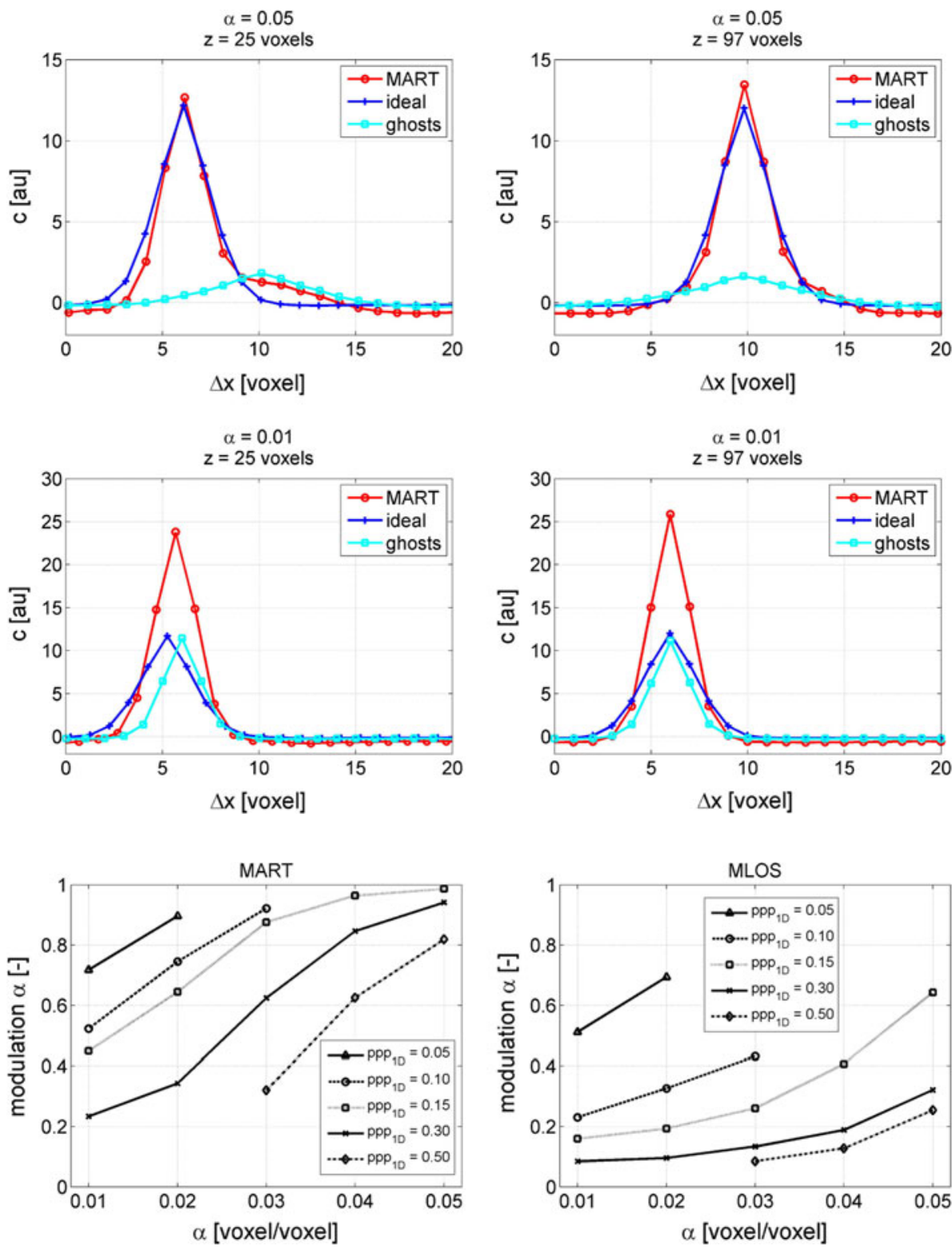

Further simulations have shown less dramatic velocity gradient bias errors for lower particle concentrations, i.e. more accurate tomographic reconstructions. These results are summarized in Fig. 8 showing the displacement gradient modulation for varying $p p p$ and displacement gradients for both MART and MLOS reconstructions. Here, the modulation is defined as the measured divided by the actual displacement gradient, where the former is obtained from a linear fit of the measured profiles such as those shown in Fig. 4. An attempt is made to collapse the modulation curves for seeding densities and displacement gradients using the reconstruction signal-to-noise ratio $N_{\mathrm{p}} / N_{\mathrm{g}}^{*}$ based on the estimated number of reoccurring ghosts, which is presented in Fig. 9. The variable $l *$ needed to compute $N_{\mathrm{p}} / N_{\mathrm{g}}^{*}$ (Eq. 5) is taken as: $l^{*}=d_{\mathrm{p}} / \alpha$. The collapse clearly is not perfect,

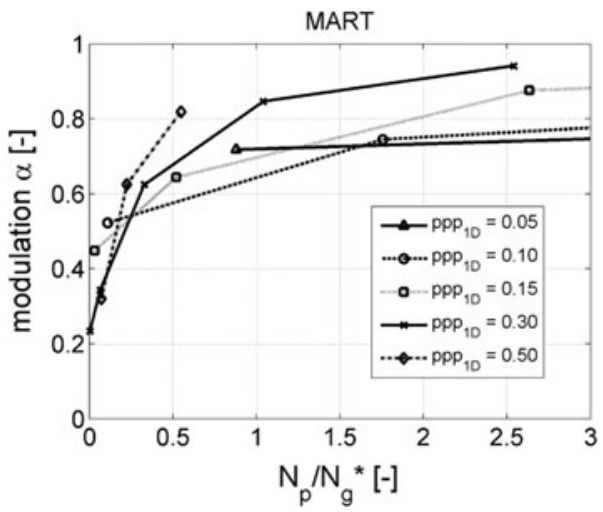

Fig. 9 Modulation of the displacement gradient $\alpha$ versus $N_{\mathrm{p}} / N_{\mathrm{g}}^{*}$ for varying 1-D particle image densities showing a reasonable collapse of the profiles in Fig. 8 for the MART reconstruction 
which can be expected given the complexity of the MART iterations, the fact that the average lower peak intensity of the ghosts has been ignored and the other simplifying assumptions in deriving $N_{\mathrm{p}} / N_{\mathrm{g}}^{*}$. Nevertheless, it can be concluded that above approximately $N_{\mathrm{p}} / N_{\mathrm{g}}^{*}=1$ velocity bias errors are relatively small and appear to decrease even with increasing particle image density. This result may be a useful criterion to avoid bias errors from ghost particles.

\subsection{Counter rotating vortices}

The effect of the reoccurring ghost particles on counter rotating vortical structures representing a wake type flow is shown in Fig. 10 for a 1-D particle image density of $0.15 \mathrm{ppp}$. The particle displacement field in these simulations is given by the superposition of individual vortices with angular voxel displacement $\xi_{\theta}$ defined as:

$\xi_{\theta}=\beta \frac{r}{2} \exp \left(-\frac{r}{R}\right)$

where $\beta$ is the peak vorticity (in voxels/voxel) evaluated at the vortex axis, $r$ is the radial distance from the axis, and $R$ is the core radius. Vorticity in this section will be based on the particle displacement gradients, rather than the velocity gradients as in its true definition. The difference is simply the time separation $\Delta t$ between the exposures. The gradients have been evaluated from the particle displacement using a second-order regression. All length scales are in voxel units.

As for the shear layer, a modulation of the particle displacement gradient is visible resulting in significantly reduced vorticity level for the case of a lower peak vorticity ( 0.03 voxel/voxel, Fig. 10-left). Please note here that the measured local flow topology, as represented by the general shape of the vorticity contours, is still in good qualitative agreement with the actual flow pattern. This is important as Tomographic-PIV is mainly used at present to investigate flow topology, i.e. coherent structures. For a large peak vorticity ( 0.20 voxel/voxel, Fig. 10-right), the actual particle displacement variation over the volume is larger so that the ghosts again decorrelate and do not affect the correlation signal significantly. Consequently, the velocity field is measured with a higher accuracy.

Profiles of the particle displacement in the $x$-direction through the vortex core (Fig. 11) reveal the same trends with increasing actual particle displacement variation over the volume depth as for the shear layer (Fig. 4). The ghost particles seem to take on the depth average velocity except for the highest peak vorticity case. There, however, the correlation signal-to-noise ratio is so small that the displacement may be affected by secondary effects, in particular the finite viewing angles.
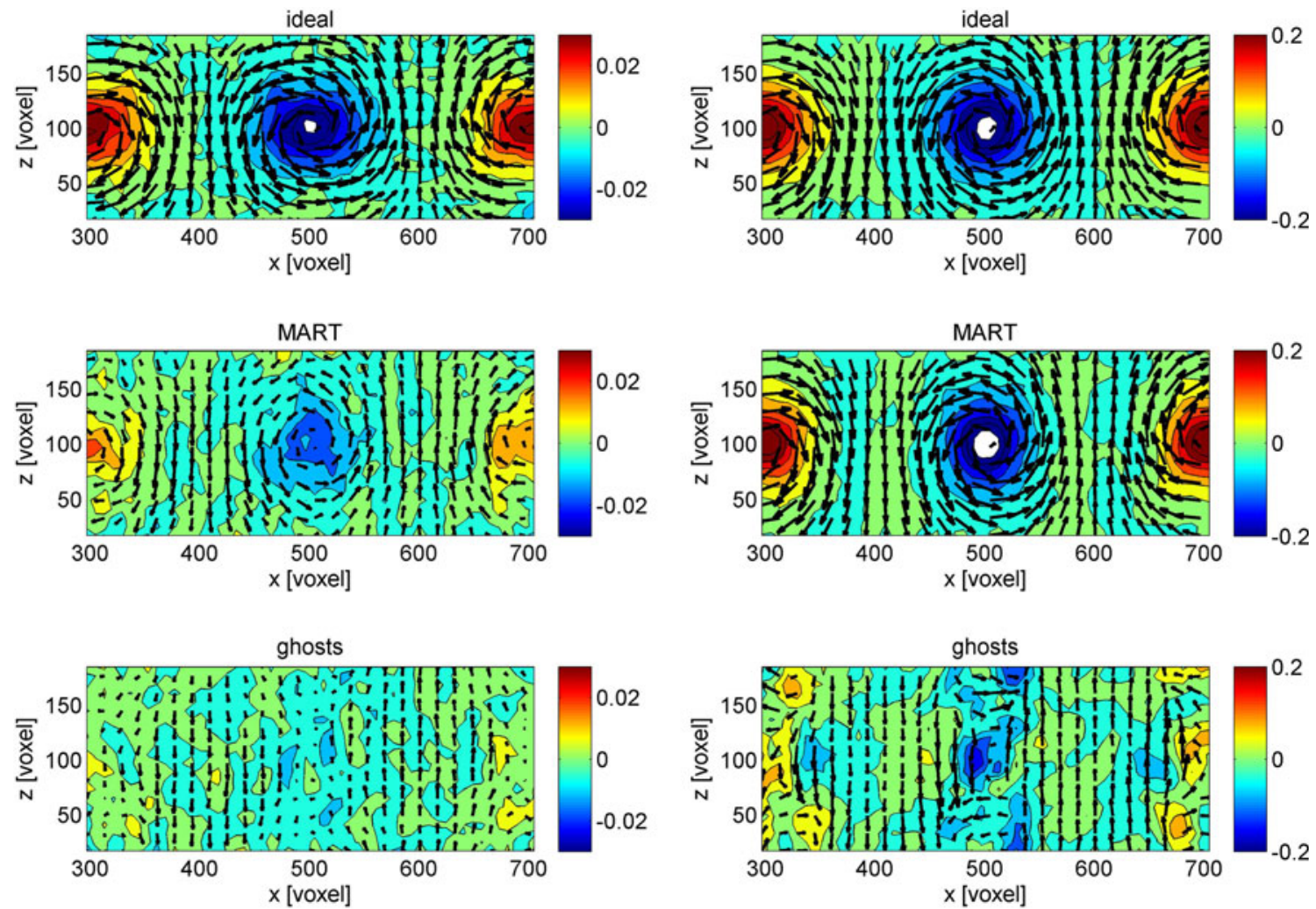

Fig. 10 The velocity (vectors) and vorticity fields (contours) for peak vorticities $\beta$ of 0.03 (left) and 0.20 voxels/voxel (right), and $p p p_{1-\mathrm{D}}=0.15$. Results are presented for the ideal reconstruction (top), MART reconstruction (middle) and ghost intensities (bottom) 
Fig. 11 Profiles of the particle displacement in the $x$-direction through the vortex core at $x=500$ voxels in a numerical simulation for peak vorticities $\beta=0.03,0.06,0.10$ and 0.20 voxels/voxel, and $p p p_{1-\mathrm{D}}=0.15$. Results are presented for MART, MLOS and ideal reconstructions together with the ghost particle velocity and they indicate a velocity gradient modulation caused by ghost particles, which decreases as the vorticity increases
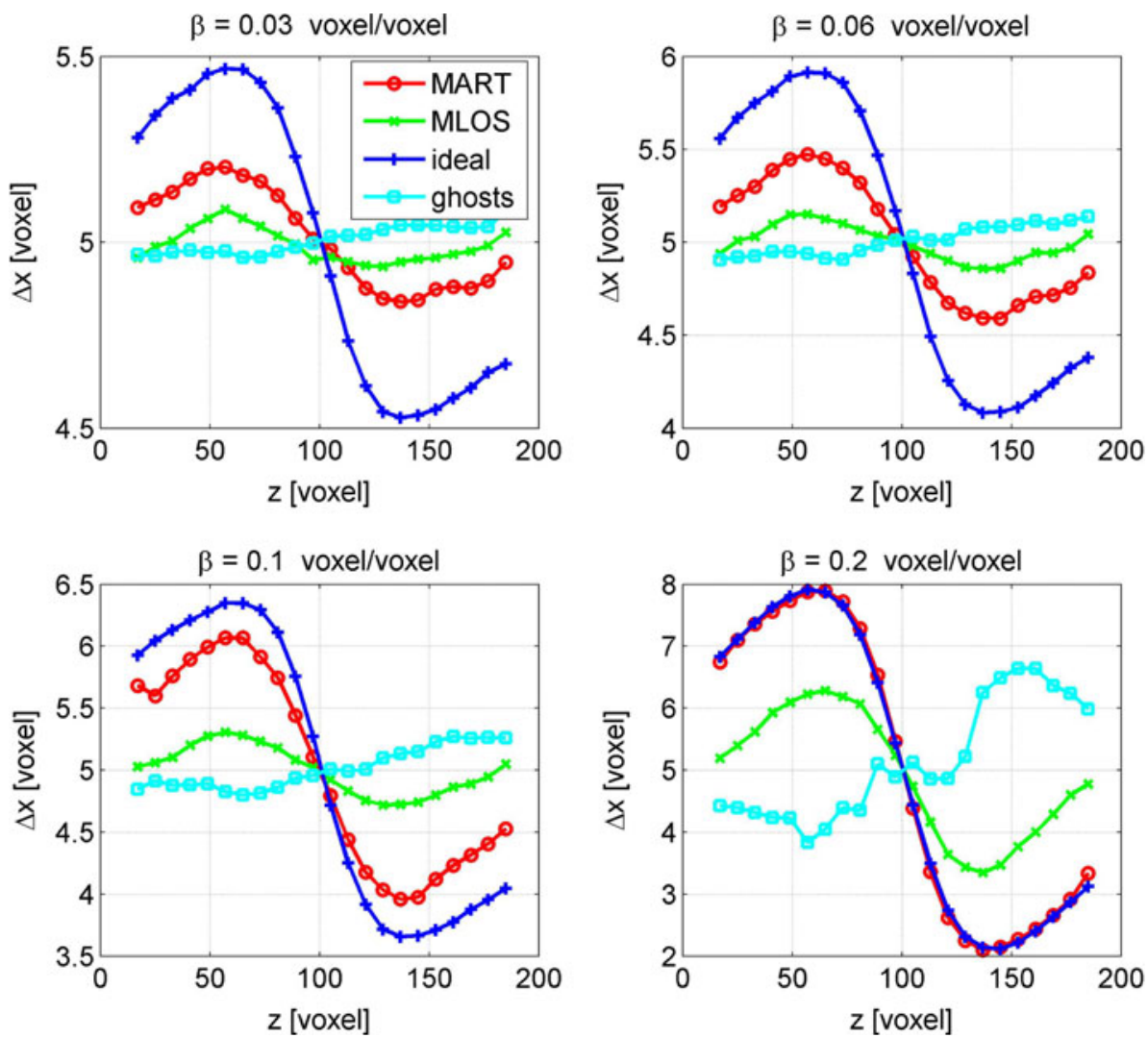

In between the vortex cores, the actual particle displacement in the $z$-direction (depth) varies very little causing the ghost particle to displace coherently in the same direction as can be seen in Fig. 10 even for relatively strong vortices. Again the measured displacement profiles reveal an important modulation (Fig. 12), which demonstrates that also the velocity component in the depth direction can be affected by biases due to the ghosts.

From the profiles of Fig. 11 (and 12), a modulation coefficient is computed as the maximum measured vorticity (or the maximum $z$-displacement difference) for MART relative to ideal reconstruction, which is shown in Fig. 13. Because the actual displacement variation through the vortex core is larger than in between cores, the modulation coefficient is much lower in the latter indicating a larger bias error.

\section{Experiments}

This part of the paper presents evidence of ghost bias errors in real measurements of a turbulent boundary layer and a validation the overall trends observed in the numerical simulations. Also, a demonstration is given on how these errors can affect the turbulence statistics. Further, a procedure is proposed to assess $N_{\mathrm{p}} / N_{\mathrm{g}}^{*}$, that is the relative occurrence of ghost pairs, in general measurements, which is illustrated for a cylinder wake flow (Sect. 5.2).

\subsection{Validation of the numerical simulations}

For the experimental validation, time-resolved boundary layer data is used (see Schröder et al. 2008b for details on the experimental arrangement). The high image recording rate in that experiment $(3 \mathrm{kHz})$ allows for a control of the displacement variation over the volume depth, while maintaining the same turbulent flow inside the measurement volume, by changing the time separation between the exposures used in the cross-correlation analysis (at increments of $0.33 \mathrm{~ms}$ ). This increment is indeed much smaller than the smallest timescales in the flow, which are reported to be approximately $11 \mathrm{~ms}$ (Schröder et al. 2008b). To recall the other main experimental details: the Reynolds number based on momentum thickness was 2,460 and the boundary layer thickness was $37 \mathrm{~mm}$. A system of six high-speed cameras $(N=6)$ was used to measure the velocity distribution in a $60 \times 60 \times 12 \mathrm{~mm}^{3}$ volume. The spatial discretization in the images and the reconstructed objects was 12 voxels $/ \mathrm{mm}$, while the particle image density was approximately $0.06 \mathrm{ppp}$. For these experimental conditions and $A_{\mathrm{p}}=3.2$ pixels (the area is obtained from the image auto-correlation), the ratio of actual particles versus ghosts is estimated at $N_{\mathrm{p}} / N_{\mathrm{g}}=7$. Even if this ratio is high, a modulation effect is still expected for very small particle displacement variation over the volume depth. Note also that the $N_{\mathrm{p}} / N_{\mathrm{g}}$ estimate is very sensitive to 
Fig. 12 Profiles of the particle displacement in the $z$-direction between vortex cores at $x=400$ voxels in a numerical simulation for peak vorticities $\beta=0.20$ and 0.30 voxels/ voxel. Results are presented for MART, MLOS, and ideal reconstructions together with the ghost particle velocity
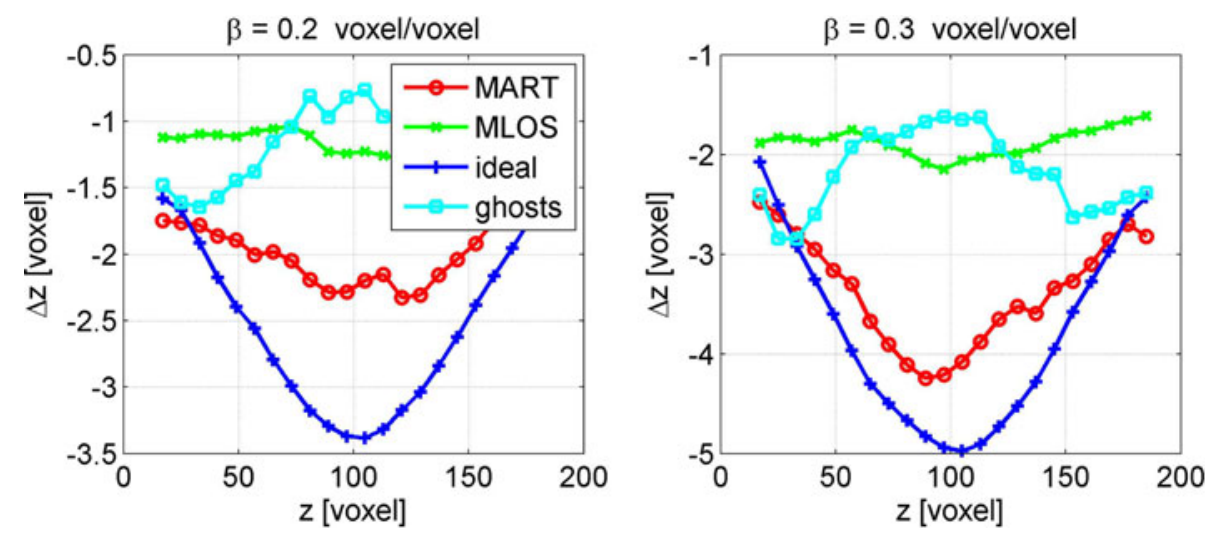

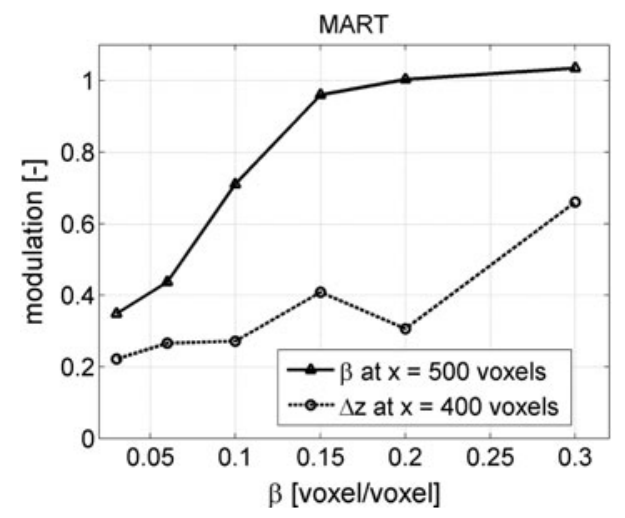

Fig. 13 Ratio of measured and actual vorticity $\beta$ (modulation) at $x=500$ voxels and the $w$-component of velocity at $x=400$ voxels versus peak vorticity $\beta$ for $p p p_{1-\mathrm{D}}=0.15$. The results are for MART reconstructions

uncertainty in the particle image density $p p p$ and effective particle image area $A_{\mathrm{p}}$ for large $N$ (Eq. 3).

In the experiment, the measurement volume depth direction, $z$, corresponds to the wall-normal direction in the flow. Therefore, the average velocity profile in $z$-direction is expected to return the mean shear in the boundary layer, similar to the above simulations of a simple, non-turbulent shear layer. However, as before, the measured profiles reveal a small velocity gradient modulation for the smallest time separation, i.e. smallest particle displacement variation ( $\Delta t=0.33 \mathrm{~ms}$, Fig. 14-left). This modulation decreases as the displacement variation increases until the profiles converge to what is considered to be the actual velocity distribution $(\Delta t=3.0$ and $4.0 \mathrm{~ms})$. These effects are most notable near the edges of the measurement volume due to the decreasing light intensity there, which increases the relative importance of the ghost particles in terms of their intensity. Based on the velocity profile for $\Delta t=4.0 \mathrm{~ms}$, the maximum difference in actual particle displacement over the volume depth is 0.25 and 3.0 voxels for the time separations $\Delta t=0.33 \mathrm{~ms}$ and $4.0 \mathrm{~ms}$, respectively. Clearly, a maximum difference of only 0.25 voxels $(\Delta t=0.33 \mathrm{~ms})$ causes the ghost particles, however, few, to reoccur in both exposures and affect the velocity measurement. On the other hand, a maximum difference of 3.0 voxels $(\Delta t=4.0 \mathrm{~ms})$ is large enough for the ghosts to de-correlate and not contribute to a velocity error.

Similarly, the accuracy of the velocity depends strongly on the number of cameras used in the tomographic reconstruction. Reducing this number from 6 to 3 cameras leads to increasing bias errors (Fig. 14-right), which is the result of a decrease in the reconstruction quality and consequently an increase in the number of ghost particle pairs (at constant time separation $\Delta t=4.0 \mathrm{~ms}$ ).

The bias error affects not only the mean but the RMS velocity fluctuations as well. However, it is not as easy to detect, because the RMS contains both signal and noise. Measurement noise is approximately constant in terms of pixels particle displacement, and therefore its relative contribution depends on the magnitude of the actual particle displacement, hence on the time separation $\Delta t$. With decreasing $\Delta t$, a larger contribution from the measurement noise is expected, which opposes the RMS reduction due to the ghosts' bias error. The auto-correlation function can be used to separate the two effects as illustrated in Fig. 15 for the $u$-component of velocity. The DC peak in the auto-correlation contains both noise and signal, but when the shift $\Delta x$ is equal or larger than an interrogation volume (the first point away from the peak in Fig. 15) the measurement noise decorrelates and only the signal remains. Hence, the signal contribution to the DC peak may be roughly estimated by extrapolating data away from the peak to $\Delta x=0$ (dashed lines). The corrected peak value is then related to the noisefree RMS fluctuations according to $u_{\mathrm{rms}}=R_{u u}(\Delta x=0)^{1 / 2}$. It is seen from Fig. 15 that the corrected RMS value and the auto-correlation around the peak increase with decreasing time separation $\Delta t$, which is consistent with the expected behaviour for the ghosts' bias error.

Further, from a visual inspection of the spatial distribution of vorticity and other local topology detection criteria (such as the $Q$-criterion, Hunt et al. 1988), it is found that main topological features of the turbulent flow are 
Fig. 14 Experimental results showing the average flow velocity over the volume depth for different separation times $\Delta t$ and using 6 cameras (left), and for different number of cameras at constant separation time $(\Delta t=4 \mathrm{~ms}$, right $)$. Increasing $\Delta t$ corresponds to increasing displacement gradient $\alpha$, hence variation of the particle displacement over the volume depth
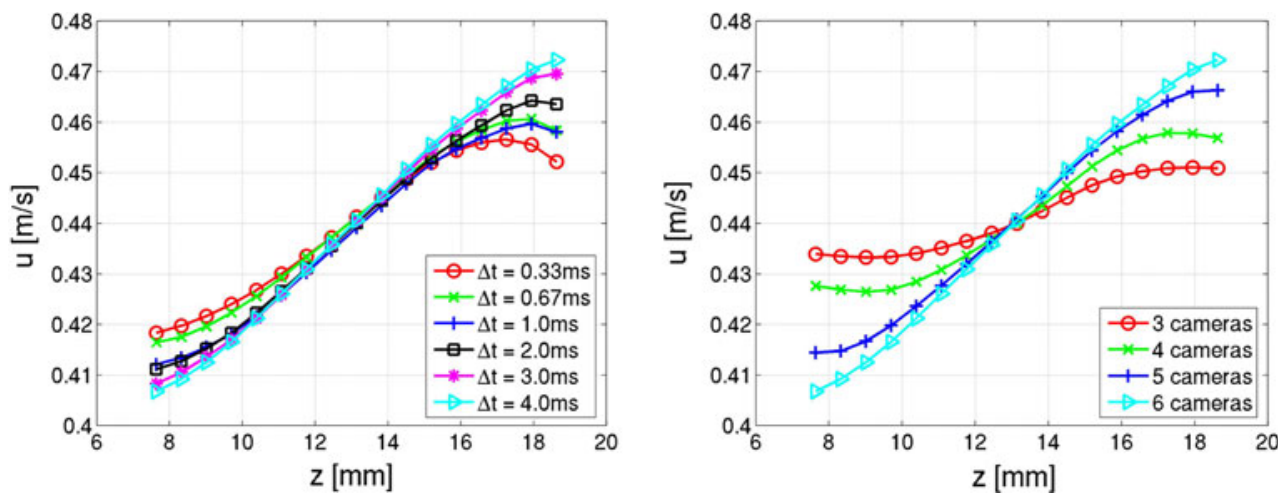

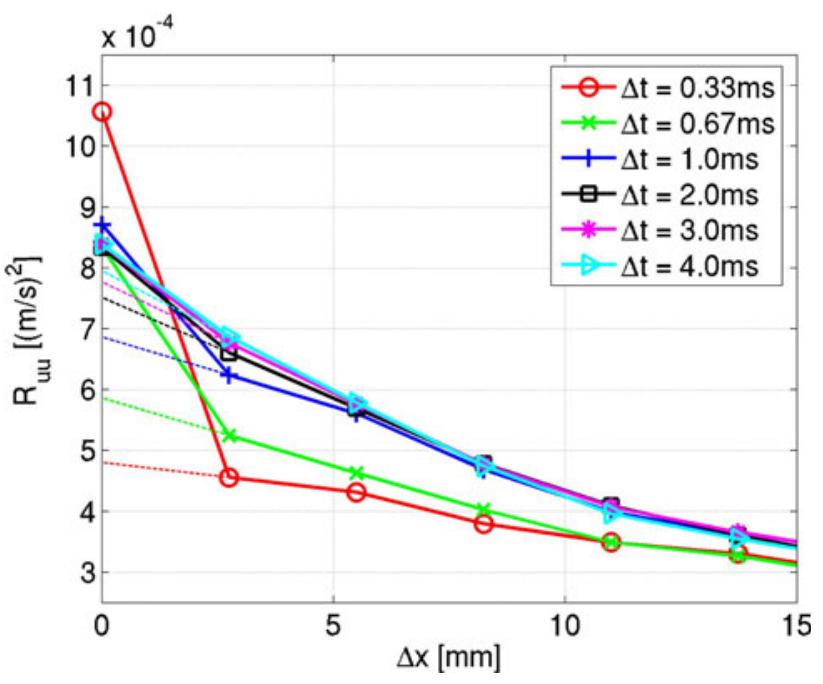

Fig. 15 Streamwise auto-correlation function for the $u$-component of velocity showing results for different separation times $\Delta t$ and 6 cameras. The data is from the wall parallel plane $z=17 \mathrm{~mm}$. The dashed lines represent values corrected for measurement noise

preserved at small $\Delta t$ (see for example Fig. 16) even though their amplitude clearly is decreased due to the bias error. At the same time, the measurement noise is increased with decreasing $\Delta t$ due to the decrease in the dynamic range of the particle displacement. For completeness, it is noted that the presented velocity gradients have been determined using a second-order regression.

The observed similarity in topology can be quantified by the correlation of the spanwise vorticity distribution $\omega_{y}$ for $\Delta t=0.33$ and $0.67 \mathrm{~ms}$ with the actual vorticity distribution, which is approximated by $\omega_{y}$ for the case $\Delta t=4.0 \mathrm{~ms}$. These correlation coefficients yield 54 and $72 \%$, respectively, which demonstrates a reasonable correspondence given the high noise level, especially at small $\Delta t$. This noise level is estimated at $40 \%(\Delta t=0.33 \mathrm{~ms})$ and $20 \%(\Delta t=0.67 \mathrm{~ms})$ based on the increased drop off in the $\omega_{y}$ auto-correlation peak w.r.t. the reference case $(\Delta t=4.0 \mathrm{~ms})$. All the above trends in the experimental results are fully consistent with the proposed model and results from numerical simulations.

\subsection{Cylinder wake revisited}

The above results can now help to understand the invariance of the velocity and vorticity statistics in the cylinder wake with changing particle image density (Elsinga et al. 2006b; Elsinga 2008), which is part of the initial motivation for this work as discussed in the introduction. Moreover, the procedure that will be outlined here may be useful in a general measurement to check whether ghost bias errors are expected to be important.

First, the variation of $N_{\mathrm{p}} / N_{\mathrm{g}}^{*}$ with the particle image density ppp needs to be established for the specific experimental conditions, i.e. the number of cameras $N=4$, the effective particle image area $A_{\mathrm{p}}=2.4$ pixels, volume thickness $l_{z}=150$ voxels, and the length scale $l^{*}$ for which the particle displacement normal to the viewing direction varies to within an effective particle image diameter $d_{\mathrm{p}}=\left(4 A_{\mathrm{p}} / \pi\right)^{0.5}=1.7$ pixels. The length $l^{*}$ can be assessed based on the measured instantaneous velocity distributions such as shown in Fig. 17-left. Suppose the accuracy of the velocity around the secondary vortices is of interest here. Then, the length $l *$ is obtained directly from a profile of the particle displacement taken along the $z$-axis and through the vortex core. In particular, the displacement component normal to the viewing direction, i.e. $z$-direction, needs to be considered (Fig. 17-right). [For simplicity the viewing direction is taken as the depth direction, $z$.] In this plot, the region where the displacement varies within $d_{\mathrm{p}}=1.7$ pixels is indicated by the horizontal blue lines. Presently, $l^{*}$ is approximately $0.5 D$, where $D \approx 150$ voxels is the cylinder diameter, which leads to $l * / l_{z}=0.5$.

Substitution of all parameters in Eq. 5 yields the result in Fig. 18, which shows $N_{\mathrm{p}} / N_{\mathrm{g}}^{*}$ for varying $p p p$ and $l * / l_{z}$. For $l * l$ $l_{z}=0.5$, it is found that $N_{\mathrm{p}} / N_{\mathrm{g}}$ * remains sufficiently large even for the largest particle image density encountered in these experiments, $p p p=0.08$. This explains why no significant differences have been observed in the results for the different particle image densities over the range $0.02<p p p<0.08$.

It should be noted that the above $l^{*}$ is specific to the core region of the secondary vortex structure, i.e. the particle 
Fig. 16 Spanwise vorticity distribution, $\omega_{y}=\partial u / \partial z-\partial w / \partial x$ $(1 / \mathrm{s})$, at the same time instant in the wall parallel plane $z=10 \mathrm{~mm}$ for time separations $\Delta t=0.67 \mathrm{~ms}$ (left) and $4.0 \mathrm{~ms}$ (right) using 6 cameras
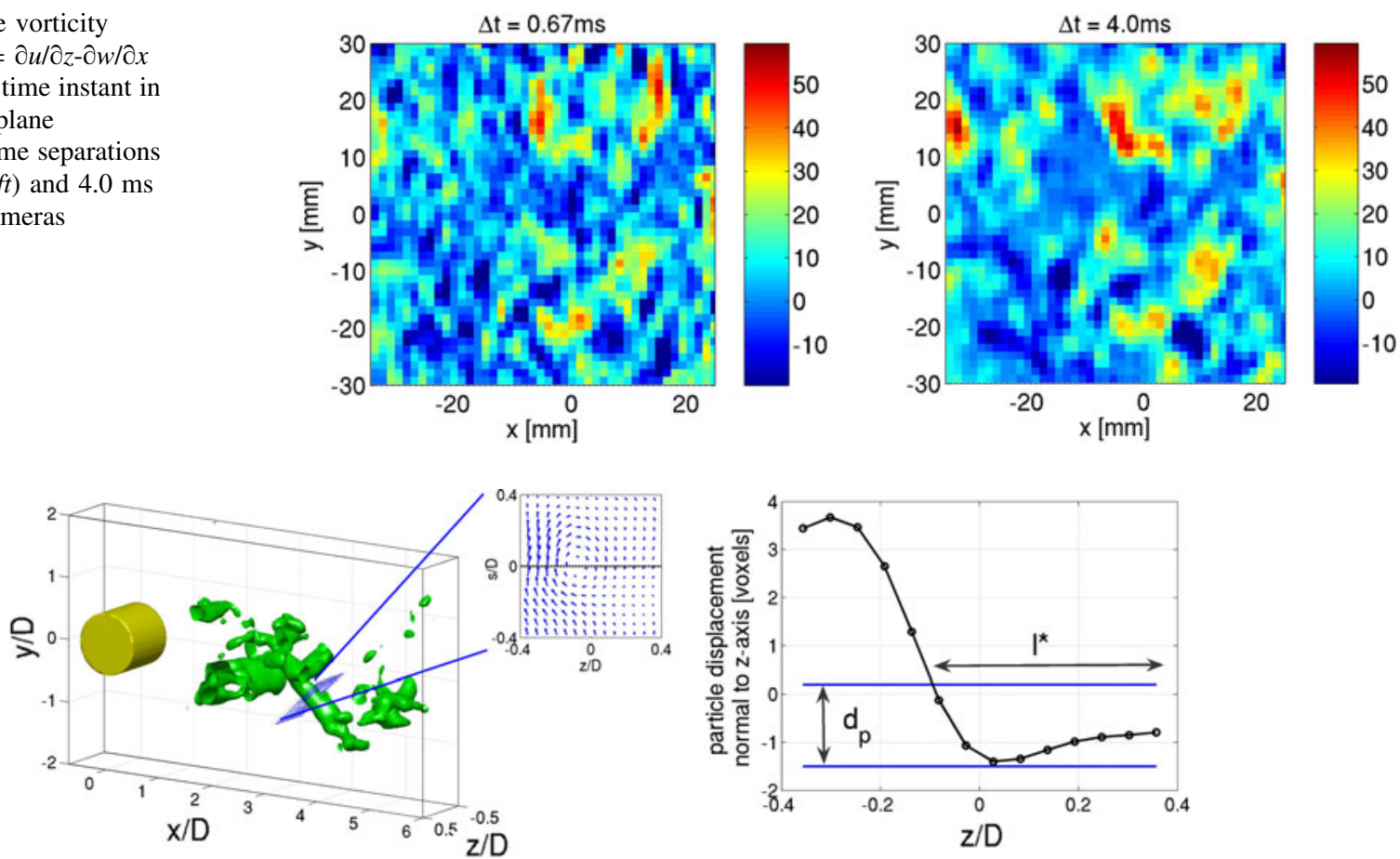

Fig. 17 Instantaneous cylinder wake showing an iso-surface of the second invariant of the velocity gradient tensor detecting vortical motion in the flow (left). Velocity vectors (blue) are plotted in a plane normal to the axis of a secondary vortex from which a profile (right)

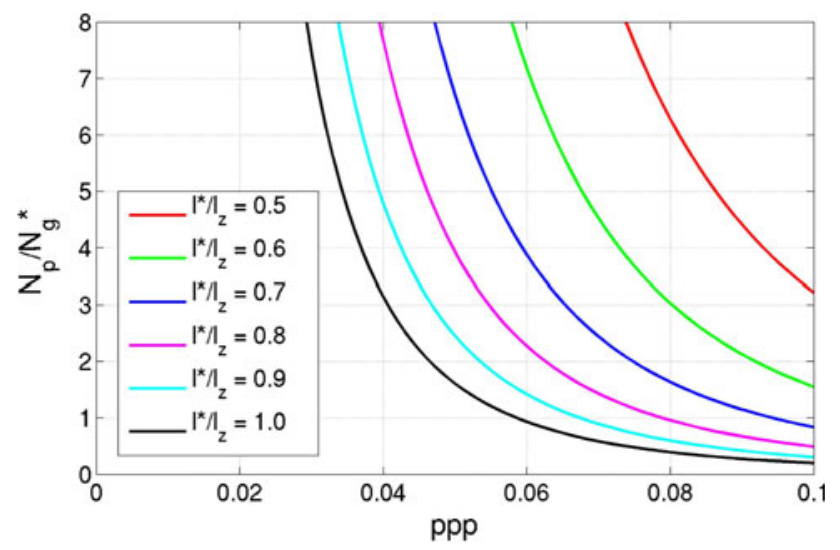

Fig. $18 N_{\mathrm{p}} / N_{\mathrm{g}} *$ as a function of particle image density ppp and $l * / l_{z}$ for the conditions in the cylinder wake experiment

displacement profile used in Fig. 17-right, and that other values may be obtained in other regions of the flow. In the outer flow region, the flow is more uniform and smaller particle displacement variations over the volume depth are expected resulting in larger values for $l^{*}$. Hence, the very small velocity gradients in these more uniform outer flow regions may be underestimated due to the ghosts, but in the absolute sense this is a small error, which is obscured by the measurement noise and uncertainty $(0.005$ voxels/voxel for the displacement gradient in this experiment, Elsinga 2008). is extracted along the line indicated. The blue horizontal lines mark the region where the variation of the particle displacement in the direction normal to the $z$-axis remains within a particle image diameter

\section{Conclusions}

The mechanism responsible for the formation of ghost particle pairs, i.e. reoccurring spurious intensity peaks, in Tomographic-PIV has been described. These reoccurring ghosts are present in both reconstructed light intensity volumes used in the cross-correlation analysis and are displaced in between by approximately the average displacement of the actual particles from which they have been formed. Therefore, they have the effect of smoothing and reducing the measured particle displacement variations (and gradients) over the volume thickness. The number of ghost pairs can be estimated from the experimental parameters as outlined in Sect. 3.

Results from numerical simulations and experiments further illustrated this phenomenon showing a decrease in the measured average shear in a shear layer and peak vorticity in vortices due to the ghost pairs. The local flow topology, however, remained largely unaffected. Furthermore, the simulations showed that the bias errors decrease with increasing reconstruction accuracy as well as with increasing actual particle displacement variation (or displacement gradient) along the line of sight, in agreement with theory. Hence, it is important to monitor the reconstruction accuracy and the displacement variation over the volume depth in real experiments. The former can be done by evaluation of the average reconstructed intensity inside and outside the laser 
sheet (Elsinga et al. 2006a; Michaelis and Wieneke 2008). A procedure to check for sufficient displacement variation has been presented in Sect. 5.2, but as a rule of thumb it can be said that the range of displacements normal to the viewing direction should exceed a particle image diameter. Alternatively, a comparison of the flow statistics with results obtained by other experimental techniques may be used to identify important bias errors. Examples of the relevant quantities in that respect are the mean velocity variation over the volume depth and the RMS velocities.

The required minimum particle displacement variation over the volume thickness can be translated into a minimum value for the time separation between (laser) light pulses without exceeding limits imposed by the crosscorrelation algorithm.

The knowledge gained on the reoccurring ghosts can further be used to improve the accuracy of the individual tomographic reconstruction (e.g. Novara et al. 2010). There are also anticipated applications related to separating ghost from actual particle trajectories in time-resolved Tomographic-PIV or 3-D PTV measurements (e.g. Schröder et al. 2008b). The ghost trajectories will be relatively short compared to the actual particle trajectories, which is due to the individual ghost particle disappearing with increasing time, i.e. increasing displacement variation of the corresponding actual particles. The actual particles, on the other hand, are never lost and can, in principle, be tracked through the entire measurement domain.

Acknowledgments The authors thank F.F.J. Schrijer for his contribution in the ensemble cross-correlation analysis. This work is supported by the Dutch Stichting FOM.

Open Access This article is distributed under the terms of the Creative Commons Attribution Noncommercial License which permits any noncommercial use, distribution, and reproduction in any medium, provided the original author(s) and source are credited.

\section{References}

Adrian RJ (1991) Particle-imaging techniques for experimental fluid mechanics. Ann Rev Fluid Mech 23:261-304

Adrian RJ, Westerweel J (2010) Particle image velocimetry. Cambridge University Press, Cambridge

Atkinson C, Soria J (2009) An efficient simultaneous reconstruction technique for tomographic particle image velocimetry. Exp Fluids 47:553-568

Atkinson C, Coudert S, Foucaut JM, Stanislas M, Soria J (2009) Thick and thin volume measurements of a turbulent boundary layer using tomographic particle image velocimetry. 8th Int Symp on PIV, Melbourne, Australia

Coëtmellec S, Buraga-Lefebvre C, Lebrun D, Özkul C (2001) Application of in-line digital holography to multiple plane velocimetry. Meas Sci Technol 12:1392-1397

Elsinga GE (2008) Tomographic particle image velocimetry and its application to turbulent boundary layers. $\mathrm{PhD}$ thesis, Delft
University of Technology, Delft, The Netherlands (http:// repository.tudelft.nl/file/1003861/379883)

Elsinga GE, Marusic I (2010) Evolution and lifetimes of flow topology in a turbulent boundary layer. Phys Fluids 22:015102

Elsinga GE, Scarano F, Wieneke B, Van Oudheusden BW (2006a) Tomographic particle image velocimetry. Exp Fluids 41:933-947

Elsinga GE, Van Oudheusden BW, Scarano F (2006b) Experimental assessment of tomographic-PIV accuracy. 13th Int Symp on Appl of Laser Techniques to Fluid Mech, Lisbon, Portugal

Elsinga GE, Kuik DJ, van Oudheusden BW, Scarano F (2007) Investigation of the three-dimensional coherent structures in a turbulent boundary layer. 45th AIAA Aerospace Sciences Meeting, Reno, NV, USA, AIAA-2007-1305

Elsinga GE, Adrian RJ, Van Oudheusden BW, Scarano F (2010) Threedimensional vortex organization in a high Reynolds number supersonic turbulent boundary layer. J Fluid Mech 644:35-60

Hain R, Kähler CJ, Michaelis D (2008) Tomographic and time resolved PIV measurements on a finite cylinder mounted on a flat plate. Exp Fluids 45:715-724

Herman GT, Lent A (1976) Iterative reconstruction algorithms. Compt Biol Med 6:273-294

Humble RA, Elsinga GE, Scarano F, Van Oudheusden BW (2009) Three-dimensional instantaneous structure of a shock wave/ turbulent boundary layer interaction. J Fluid Mech 622:33-62

Hunt JCR, Wray AA, Moin P (1988) Eddies, stream, and convergence zones in turbulent flows. Cent Turbul Rep CTR 88:193-208

Kühn M, Ehrenfried K, Bosbach J, Wagner C (2009) Application and validation of large scale tomographic PIV in a long rectangular convection cell. 8th Int Symp on PIV, Melbourne, Australia

Louis AK (1981) Ghosts in tomography-the null space of the radon transform. Math Meth Appl Sci 3:1-10

Maas HG, Gruen A, Papantoniou D (1993) Particle tracking velocimetry in three-dimensional flows. Exp Fluids 15:133-146

Meinhart CD, Wereley ST, Santiago JG (2000) A PIV algorithm for estimating time-average velocity fields. J Fluids Eng 122:285-289

Michaelis D, Wieneke B (2008) Comparison between Tomographic PIV and Stereo PIV. 14th Int Symp on Appl of Laser Techniques to Fluid Mech, Lisbon, Portugal

Munshi P (2002) Picking the right solution from a set of correct solutions. Meas Sci Technol 13:647-653

Natterer F (1986) The mathematics of computerized tomography. Wiley, New York

Novara M, Batenburg KJ, Scarano F (2010) Motion trackingenhanced MART for tomographic PIV. Meas Sci Technol 21:035401

Ortiz-Duenas C, Kim J, Longmire E (2010) Investigation of liquidliquid drop coalescence using tomographic PIV. Exp Fluids online. doi:10.1007/s00348-009-0810-7

Raffel M, Willert CE, Kompenhans J (1998) Particle image velocimetry: a practical guide. Springer, Berlin

Scarano F, Riethmuller ML (2000) Advances in iterative multigrid PIV image processing. Exp Fluids Suppl 29:S51-S60

Schröder A, Geisler R, Elsinga GE, Scarano F, Dierksheide U (2008a) Investigation of a turbulent spot and a tripped turbulent boundary layer flow using time-resolved tomographic PIV. Exp Fluids 44:305-316

Schröder A, Geisler R, Staack K, Wieneke B, Elsinga GE, Scarano F, Henning A (2008b) Lagrangian and Eulerian views into a turbulent boundary layer flow using time-resolved tomographicPIV. 14th Int Symp on Appl of Laser Techniques to Fluid Mech, Lisbon, Portugal, submitted to Exp Fluids

Wieneke B (2008) Volume self-calibration for 3D particle image velocimetry. Exp Fluids 45:549-556

Worth NA, Nickels TB (2008) Acceleration of Tomo-PIV by estimating the initial volume intensity distribution. Exp Fluids $45: 847-856$ 